\title{
The use of morphokinetic parameters to select all embryos with full capacity to implant
}

\author{
Sandrine Chamayou • Pasquale Patrizio • \\ Giorgia Storaci - Venera Tomaselli • Carmelita Alecci • \\ Carmen Ragolia • Claudia Crescenzo • \\ Antonino Guglielmino
}

Received: 3 January 2013 / Accepted: 28 March 2013 / Published online: 13 April 2013

(C) The Author(s) 2013. This article is published with open access at Springerlink.com

\begin{abstract}
Purpose Embryo kinetics analysis is an emerging tool for selecting embryo(s) for transfer. The aim of the present study was to determine morphokinetic parameters easily usable in the laboratory and predictive of embryo development and, most importantly, of embryo competence in producing a clinical pregnancy after day 5 transfer.

Methods A retrospective time-lapse monitoring analysis of morphokinetic parameters for 72 fully implanted embryos (group A) were compared to 106 non-implanted embryos (group B), and to 66 embryos with arrested development from the same pool of group A. All the embryos were from 78 patients undergoing ICSI treatment and day 5 embryo transfers. Results A day 3 embryo will develop into a viable blastocyst if the following ranges of morphokinetic parameters are met: $\mathrm{t} 1$ (between $18.4 \mathrm{~h}$ and $30.9 \mathrm{~h}$ post-ICSI), $\mathrm{t} 2$ (21.4-34.8 $\mathrm{h}$ ), $\mathrm{t} 4$ (33.1$57.2 \mathrm{~h}), \mathrm{t} 7$ (46.1-82.5 h), t8 (46.4-97.8 h), tC-tF (7.7-22.9 h) and s3 (0.7-30.8 h). On day 5 embryos with the highest probability to implant are those with a cc3 between $9.7 \mathrm{~h}$ and $21 \mathrm{~h}$.
\end{abstract}

Capsule Embryos were cultured until day 5 and time-lapse monitored. We defined a methodology applicable for any laboratory to determine in its conditions of work which parameters are predictive of embryo competence to develop into a viable embryo on day 5 and to give a pregnancy when transferred.

S. Chamayou $(\bowtie) \cdot$ G. Storaci $\cdot$ C. Alecci $\cdot$ C. Ragolia $\cdot$

C. Crescenzo $\cdot$ A. Guglielmino

Unità di Medicina della Riproduzione, Fondazione HERA,

Via Barriera del Bosco n. 51/53,

95030 Sant'Agata Li Battiati, CT, Italy

e-mail: s.chamayou@yahoo.fr

\section{P. Patrizio}

Yale University Fertility Centre, 150 Sargent Drive,

New Haven, CT 06511, USA

V. Tomaselli

Department of Political and Social Sciences, University of Catania,

Catania, Italy
Conclusions Morphokinetic parameters are helpful to make appropriate decisions for the disposition of each embryo. It is recommended that each laboratory should determine its own ranges of in vitro development (IVD-MKP) and implantationassociated (IMP-MKP) morphokinetic parameters.

Keywords Development · Embryo competence .

Embryo kinetic $\cdot$ Morphokinetic parameter $\cdot$ Morphology ·

Time-lapse monitoring

\section{Introduction}

It is well known that morphological criteria alone during the first days of embryo culture are poorly predictive of both embryo development and ability to procure a pregnancy $[7,11]$. However, despite these limitations embryos are still classified and chosen for transfer according to their morphological and developmental scores [1]. Embryos deemed most suitable for transfer are generally those that display precise evolutions of their in vitro growth observed at fixed times: fertilization (observation of two pronuclei) at between 16 and $18 \mathrm{~h}$ post insemination: syngamy at $23 \mathrm{~h}( \pm 1 \mathrm{~h})$; early cleavage ( $26 \mathrm{~h}$ post ICSI and $28 \pm 1 \mathrm{~h}$ post IVF); day 2 cleavage at $44 \mathrm{~h}$ ( $\pm 1 \mathrm{~h})$; day 3 cleavage ( 8 cells stage) at $68 \mathrm{~h}( \pm 1 \mathrm{~h})$; morula stage at $92 \mathrm{~h}( \pm 2 \mathrm{~h})$, and blastocyst stage at $116 \mathrm{~h}( \pm 2 \mathrm{~h})$. These standard checkpoints are not informative of particular cellular events nor of the precise kinetic of embryo development occurring between two microscopic observations. Furthermore, with the conventional embryo assessment, the embryos must be removed from the incubator and thus exposed to variations of temperature and $\mathrm{pH}$ of culture medium. Theoretically, by knowing the precise kinetic of development for each embryo the choice of embryos for transfer or for further in vitro culture should become more accurate. 
Several systems for time-lapse monitoring have been proposed as non-invasive methods for identifying embryo competence. Many articles have described the technology of continuous embryo monitoring as harmless both in animal models and humans $[2,5,12,15]$ by reporting the kinetic of human embryo development in the first days [13]. Continuous monitoring systems have described morphokinetic parameters predictive of: a) the competence of the embryos to implant when transferred within the first three days of in vitro culture $[3,10]$ and $b)$ the consequences of in vitro culture conditions on embryo kinetics $[4,6,8]-$ See for review [17]. On a global view, it is known that the gene expression leads the embryo kinetic [16] but embryo kinetic itself depends also on environmental conditions [15].

In most of the studies published until now, the authors defined one or several specific parameters as time limit (mean or median and standard deviation according to statistical analyses) against which the competence of the embryo to continue its development in vitro or to implant is predicted. The application of those calculated time limits (mean/median, \pm standard deviation) exclude for the clinical use those embryos with kinetic data out of the calculated ranges but which still have the capacity to develop and implant.

The objective of the present study is to report the observed extended time intervals of morpho-kinetic parameters that can still predict the competence of the embryo, from the early cleavage stage, to develop into a viable embryo on day 5 (in vitro development-morphokinetic parameters, IVD-MKP) and to give a clinical pregnancy when transferred (implantation-morphokinetic parameters, IMP-MKP).

The use of time intervals instead of a fixed time limit add flexibility to the busy laboratory routines and embryos within or outside the established time intervals can be properly assigned to the clinical use.

\section{Material and methods}

Population, ICSI and time-lapse culture

Prior to enrollment, all patients $(n=78)$ signed a written consent allowing the use of their data for research purposes. All patients underwent ICSI treatment with fresh oocytes and fresh ejaculated spermatozoa (with concentrations $\geq 1$ million $/ \mathrm{ml}$ ). Infertility due to severe endometriosis, premature ovarian failure and severe asthenoteratozoospermia were excluded from the study. The ovarian stimulation was carried out by the administration of luteal gonadotrophin-releasing hormone analogue (GnRHa) (Suprefact: Hoechst Marion Roussel Deutschland GmbH, Frankfurt, Germany) followed by recombinant FSH (Gonal-F: Merck-Serono, London, UK or Puregon, MSD, Franklin Lakes, USA) from cycle day 3. Vaginal ultrasound-guided aspiration of oocyte-cumulus complexes was performed $35 \mathrm{~h}$ after human chorionic gonadotrophin administration (HCG 10,000 IU, Gonasi: AMSA, Italy). Oocyte denudation and ICSI were performed $3 \mathrm{~h}$ after retrieval and the in vitro culture was carried out in $25 \mathrm{ul}$ of HTF cleavage Quinn's medium (SAGE, Trumbull, USA) under mineral oil until day 3 (4-8 cells stage) in automated incubators with $5 \% \mathrm{CO} 2,5 \% \mathrm{O} 2$ at $37{ }^{\circ} \mathrm{C}$ fitted with timelapse image acquisition (Embryoscope, Unisense, Aarhus Denmark). Seventy-two hours from ICSI the in vitro culture media was changed to HTF blastocyst Quinn's medium (SAGE). During incubation in the Embryoscope, seven plane focal images were generated each $20 \mathrm{~min}$ and recorded.

The patients were divided into two groups: Group A comprised 35 patients, aged between 27 and 40 years old (mean age: $33 \mathrm{y} \pm 3.9)$. Their embryos $(n=138)$ were divided in two subgroups: A-Imp embryos $(n=72)$ all implanted $(100 \%)$ after day 5 transfer and all resulted in clinical pregnancies (visualization of fetal heartbeat) and A-NU $(n=66)$ non usable embryos, meaning that these embryos could not be used for cryopreservation nor transfer because of impaired in vitro development (59/66 arrested prior to reaching blastocyst stage and 7/66 arrested at early blastocyst stage, after $144 \mathrm{~h}$ of in vitro culture). The 72 A-Imp embryos were transferred as follows: one patient had a single embryo-transferred, 31 patients had two embryos-transferred and 3 patients had three embryos transferred as they requested in the written consent.

A triplet pregnancy naturally reduced to a twin pregnancy at the 18th week of gestation. The single pregnancy and eight twin pregnancies are currently still on-going. The other 25 pregnancies concluded with the birth of healthy babies in number corresponding to the number of transferred and implanted embryos.

The group B comprised 43 patients, aged between 26 and 40 years old (mean age: $34 \mathrm{y} \pm 4.2$ ). A total of 106 embryos were transferred on day 5 and none implanted (all negative pregnancy tests). The 106 embryos were transferred as follows: 23 patients had two embryos transferred and 20 patients had three embryos transferred.

The in vitro culture was carried out for about $116 \mathrm{~h}$ for groups A-Imp and B, and $144 \mathrm{~h}$ for group A-NU. Embryo kinetics were recorded and studied for each embryo in all groups.

The blastocysts were scored as established into the ASRM and ESHRE consortium [1].

\section{Definition of kinetic parameters}

The time $\mathrm{t} 0$ was defined as the time of ICSI. The $\mathrm{tF}$ was defined as the time of the first frame in which both pronuclei could be observed. The time $\mathrm{tC}$ was the frame with the last observation of both pronuclei. From this time, the nucleolus precursor bodies decreased in size and subsequently disappeared, followed by pronuclei membrane flattening. The successive frame corresponded to one cell stage and it 
was annotated as $\mathrm{t} 1$. The $\mathrm{t} 2, \mathrm{t} 3, \mathrm{t} 4, \mathrm{t} 5, \mathrm{t} 6, \mathrm{t} 7, \mathrm{t} 8$ and $\mathrm{t} 9$ were defined as the times for the corresponding number of cells ( $\mathrm{t} 2$ for 2 cells, $\mathrm{t} 3$ for 3 cells, etc.). The times were annotated at the first frame in which the cells (blastomeres) were seen as separated by individual membranes. The time tM was defined as the first frame in which the embryos were compacting into the morula stage. The tB time was defined as the frame in which a crescent-shaped area began to emerge from the morula. The successive frame was the time tEB of expanded blastocyst, consistent with the increase of the overall volume of the embryo and expansion of the blastocoele cavity.

The second round of cleavage was defined $\mathrm{cc} 2$ and it was calculated as $\mathrm{t} 3-\mathrm{t} 2$; the third round of cleavage was defined cc3 and it was calculated as $15-\mathrm{t} 3$; the fourth round of cleavage was defined as cc4 and it was calculated as $\mathrm{t} 9-\mathrm{t} 5$.

An additional parameter assessed was the synchronization of cell division(s), i.e. the time required for each blastomere to replicate and reach the successive cell cycle. The synchronization parameters were defined as s2 (calculated as t4-t3) and $\mathrm{s} 3$ (calculated as $\mathrm{t} 8 \mathrm{-t}$ ). The difference $\mathrm{tC}-\mathrm{tF}$ was the duration of time for visible pronuclei. All times were expressed in hours and fraction of an hour. The morphokinetic parameters and calculated durations are illustrated in Fig. 1.

\section{Statistical analysis}

All analyzed data were continuous variables. The hypothesis about distributions of post-ICSI timings and calculated timings (cc2, cc3, cc4, s2 and s3) of embryos from group AImp and A-NU, and group A-Imp and B were tested by the two-sample Kolmogorov-Smirnov non-parametric test. In both cases, each group was taken as an independent sample.
The means of samples with normal distribution and of sufficient size were compared by ANOVA parametric test. In case of non-normal distribution, the medians were compared by Kruskall-Wallis non-parametric test.

The $Z$-test was used to establish different significance levels $(p<0.05 ;<0.01 ;<0.001)$.

\section{Results}

Embryo development

The means, medians and standard deviations of embryos from groups A-Imp, A-NU and B are given and compared in Tables 1 and 2.

\section{Embryo morphology}

In group A-Imp, all transferred embryos $(n=72)$ implanted. At the time of the embryo-transfer, $10 \%$ (7/72), $31 \%$ (22/72) and $60 \%(43 / 72)$ of the embryos were at morula, blastocysts and expanded blastocyst stage (grade 3 according to ASRM and ESHRE consensus, [1]) respectively.

In group $\mathrm{B}$, none of the transferred embryos implanted $(0 \%)$. At the time of the embryo transfers, $9 \%(10 / 106)$, $40 \%(42 / 106), 32 \%(34 / 106)$ and $19 \%(20 / 106)$ of the transferred embryos were respectively at $9+$ cells, morula, blastocyst and expanded blastocyst stages. Of note, in all the 43 embryo-transfers, at least one blastocyst was transferred.

In group A-NU, non usable embryos from the same batch of group A, $100 \%$ of embryos reached the 3 cell-stage; $94 \%(62 / 66 ; p<0.05$ compared with group A-Imp) reached

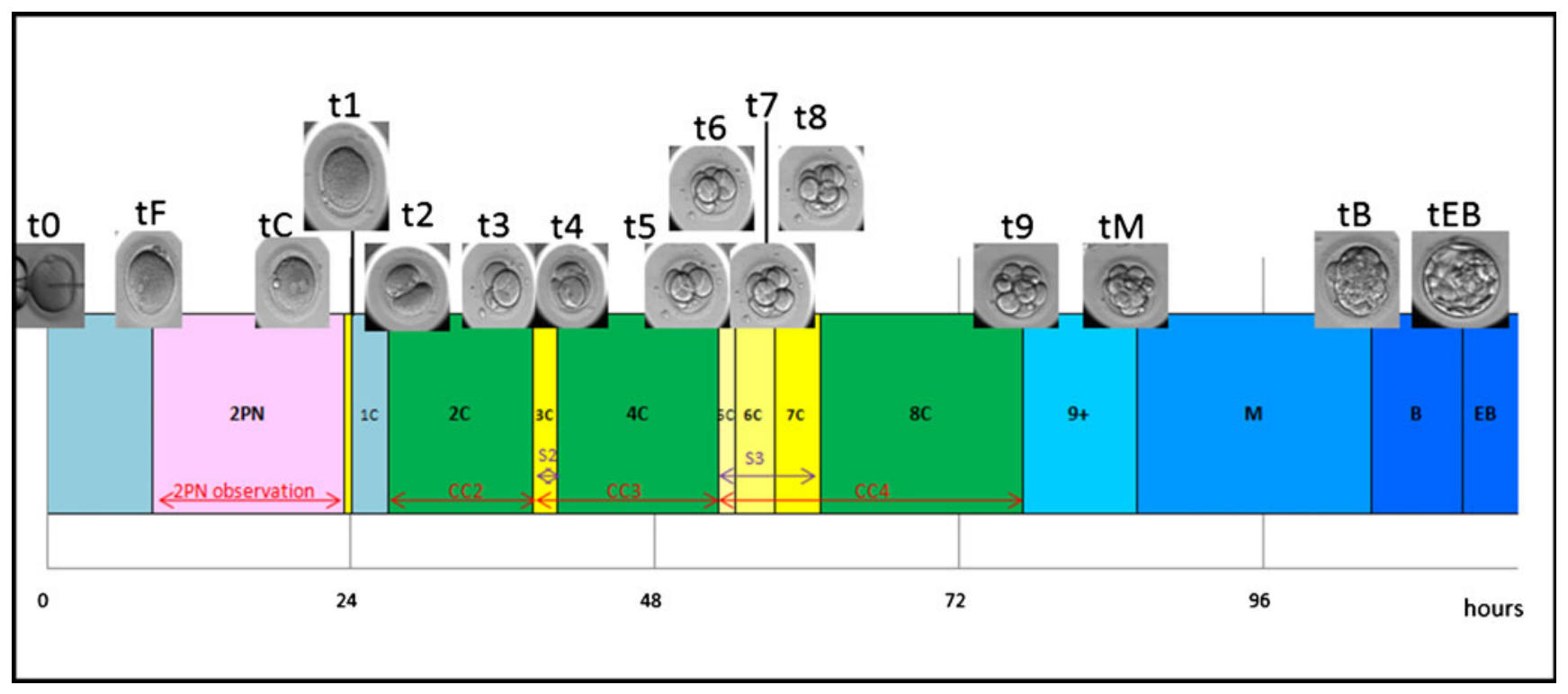

Fig. 1 Morphokinetic parameters from to to tEB (2PN: two pronuclei; $1 \mathrm{C}$ : 1 cell-stage,..., $9+$ : 9 cell-stage or more separated cells by individual membranes, M:morula, B:Blastocyst, EB: expanded blastocyst) 


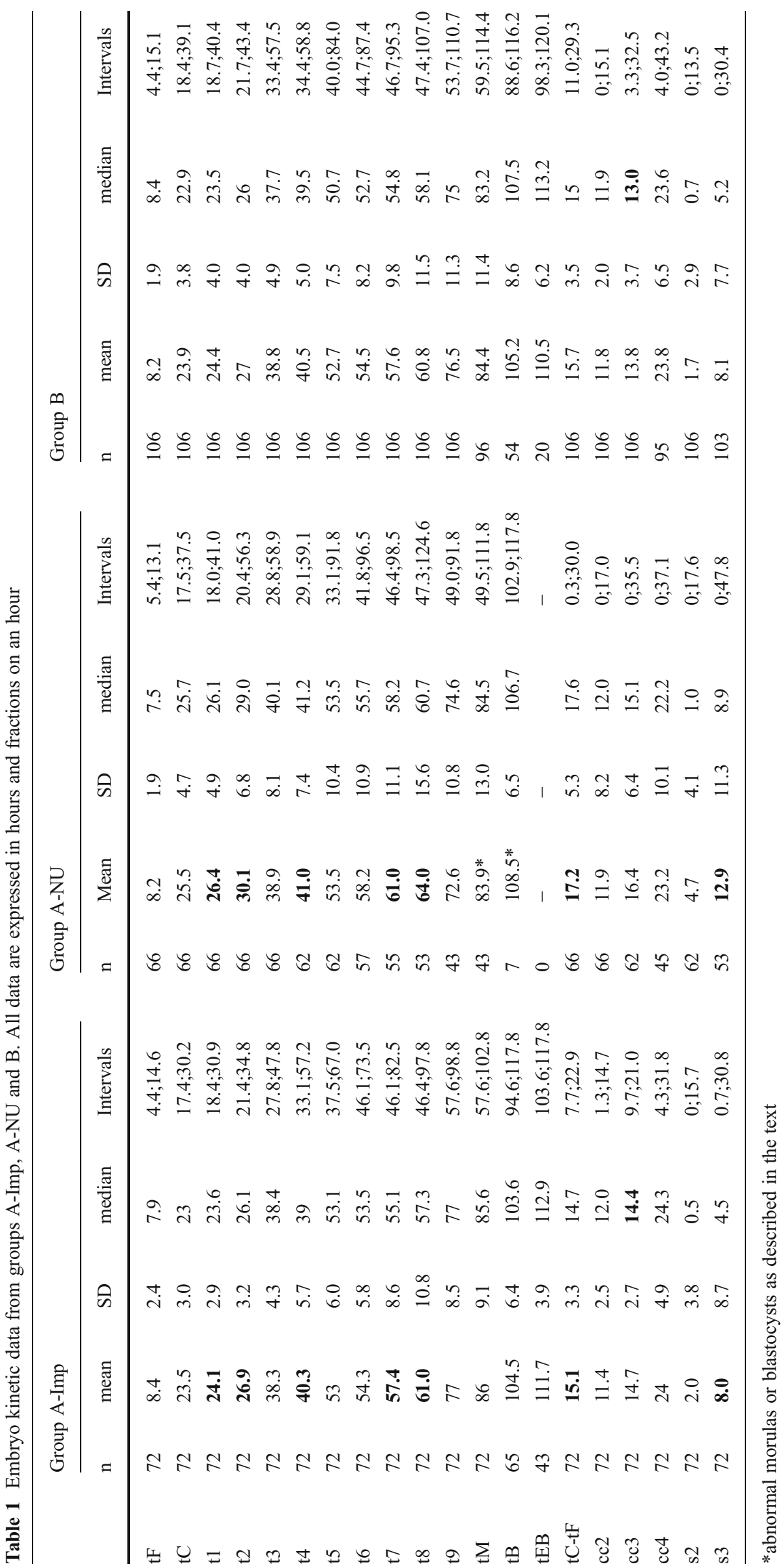


Table 2 Statistical analysis comparing the groups A-Imp with A-NU and $\mathrm{B}$

\begin{tabular}{|c|c|c|c|c|c|c|}
\hline & \multicolumn{3}{|c|}{ Groups A-Imp and B } & \multicolumn{3}{|c|}{ Groups AImp and A-NU } \\
\hline & $p 1$ & $p 2$ & $p 3$ & $p 1$ & $p 2$ & $p 3$ \\
\hline $\mathrm{tF}$ & 0.202 & 0.58 & & 0.157 & 0.076 & \\
\hline $\mathrm{tC}$ & 0.881 & 0.414 & & 0.026 & & 0.531 \\
\hline $\mathrm{t} 1$ & 0.694 & 0.580 & & 0.005 & & 0.007 \\
\hline $\mathrm{t} 2$ & 0.778 & 0.782 & & 0.012 & & 0.020 \\
\hline $\mathrm{t} 3$ & 0.366 & 0.458 & & 0.090 & 0.063 & \\
\hline t4 & 0.665 & 0.825 & & 0.003 & & 0.035 \\
\hline t5 & 0.005 & - & 0.106 & 0.593 & 0.386 & \\
\hline t6 & 0.310 & 0.856 & & 0.017 & & 0.062 \\
\hline $\mathrm{t} 7$ & 0.584 & 0.894 & & 0.006 & & 0.010 \\
\hline t8 & 0.599 & 0.922 & & 0.023 & & 0.016 \\
\hline t9 & 0.190 & 0.771 & & 0.107 & 0.178 & \\
\hline $\mathrm{tM}$ & 0.167 & 0.343 & & 0.503 & 0.301 & \\
\hline $\mathrm{tB}$ & 0.074 & 0.602 & & 0.088 & 0.095 & \\
\hline tEB & 0.621 & 0.368 & & & & \\
\hline $\mathrm{tC}-\mathrm{tF}$ & 0.306 & 0.238 & & 0.016 & & 0.032 \\
\hline $\mathrm{cc} 2$ & 0.423 & 0.268 & & 0.294 & 0.224 & \\
\hline $\operatorname{cc} 3$ & 0.001 & - & 0.004 & 0.169 & 0.610 & \\
\hline $\operatorname{cc} 4$ & 0.54 & 0.846 & & 0.012 & & 0.070 \\
\hline $\mathrm{s} 2$ & 0.016 & - & 0.100 & 0.193 & 0.628 & \\
\hline s3 & 0.092 & 0.902 & & 0.001 & & 0.010 \\
\hline
\end{tabular}

$D S$ standard deviation; $p 1$ : Kolmogorov-Smirnov test; $p 2$ : ANOVA test; $p 3$ : Kruskall-Wallis test
5 cells stage; $86 \%(57 / 66 ; p<0.001)$ reached 6 cells stage; $83 \%(55 / 66 ; p<0.001)$ reached 7 cells stage; $80 \%$ $(53 / 66 ; p<0.001)$ reached 8 cells stage; $65 \%(43 / 66$; $p<0.001)$ reached morula stage; and $11 \%(7 / 66 ; p<0.001)$ reached blastocyst stage. No embryo reached the expanding blastocyst stage. The few embryos that reached the morula or blastocyst stage were not suitable for transfer or cryopreservation because had arrested at morula stage or very early blastocyst stage as verified after $144 \mathrm{~h}$ of in vitro culture.

Embryo kinetic

\section{Comparison between group A-Imp and A-NU}

The timings (tF, t3, t5, t9, tM and $\mathrm{tB})$ and the calculated timings (cc2, cc3 and s2) between the group A-Imp and A-NU were normally distributed (Kolmogorov-Smirnov non parametric test). The distribution of the timings $\mathrm{tC}, \mathrm{t} 1$, $\mathrm{t} 2, \mathrm{t} 4, \mathrm{t} 6, \mathrm{t} 7, \mathrm{t} 8$, and the calculated timings $\mathrm{tC}-\mathrm{tF}, \mathrm{cc} 4$ and $\mathrm{s} 3$ were not normally distributed ( $p$-value $<0.05$ ). The means of the variables with normal distribution between A-Imp and ANU were not statistically different ( $p$-value $>0.05$, ANOVA test). The non-parametric Kruskall-Wallis showed no significant differences for the variables t6 and cc4 ( $p$-value $>0.05$ ), but significant differences $(p<0.05)$ for the variables $\mathrm{t} 1, \mathrm{t} 2, \mathrm{t} 4$, $\mathrm{t} 7, \mathrm{t} 8$ and the calculated timings $\mathrm{tC}-\mathrm{tF}$ and $\mathrm{s} 3$.

The morphokinetic parameters informative for embryo competence to develop in vitro (IVD-MKPs) into viable blastocysts on day 5 are shown in Fig. 2.

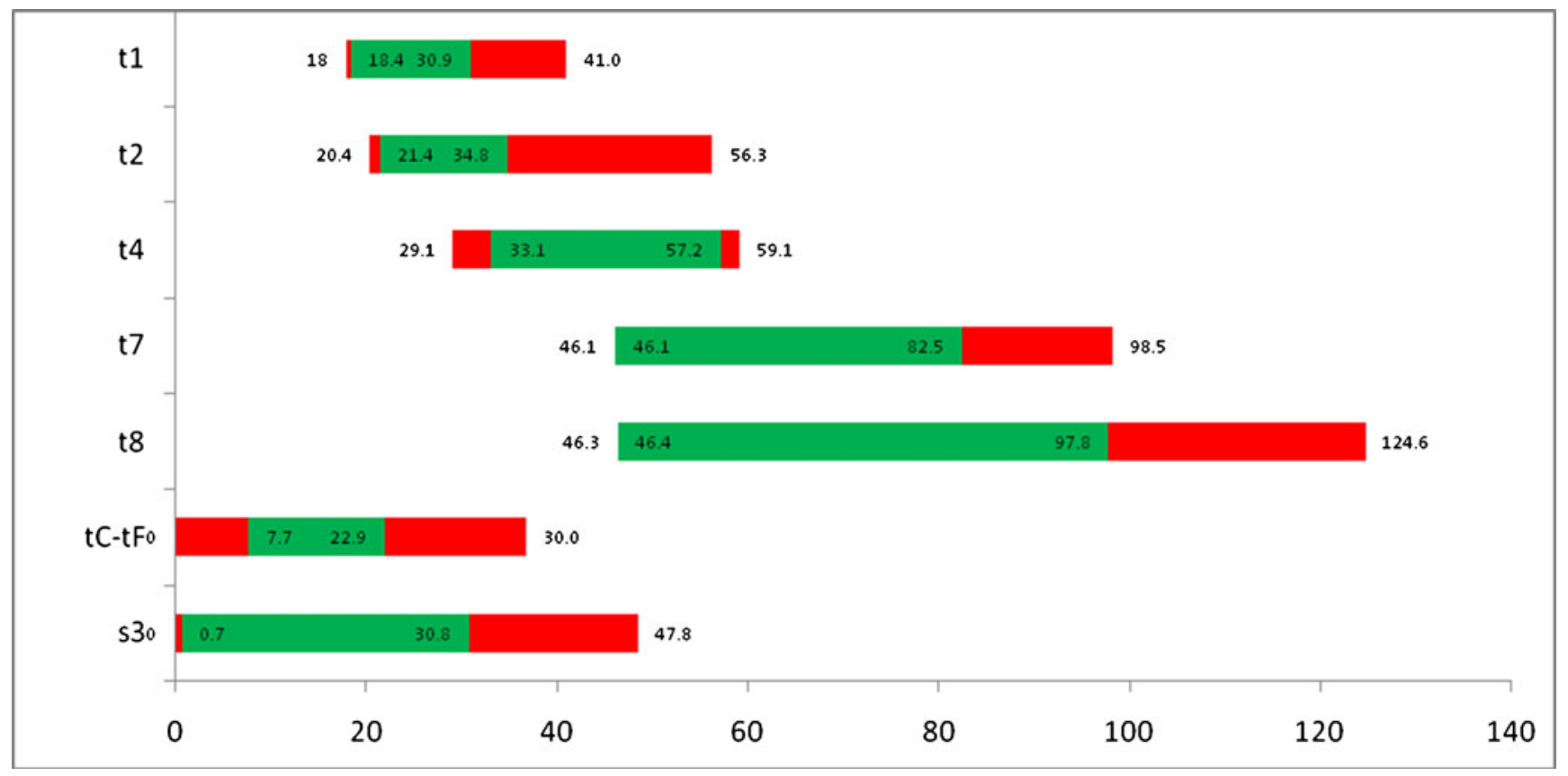

Fig. 2 Embryonic kinetic parameters determining the capacity of an embryo to become a viable embryo on the fifth day of in vitro culture (group A-Imp: green) compared with embryos with impaired development (group A-NU : red). The kinetic parameters are expressed in hour and fraction of an hour 


\section{Comparison between group A-Imp and B}

The timings ( $\mathrm{tF}, \mathrm{tC}, \mathrm{t} 1, \mathrm{t} 2, \mathrm{t} 3, \mathrm{t} 4, \mathrm{t} 6, \mathrm{t} 7, \mathrm{t} 8, \mathrm{t} 9, \mathrm{tM}, \mathrm{tB}$ and $\mathrm{tEB}$ and calculated timings ( $\mathrm{tC}-\mathrm{tF}, \mathrm{cc} 2, \mathrm{cc} 4$ and $\mathrm{s} 3$ ) between the groups A-Imp and B were normally distributed (Kolmogorov-Smirnov non-parametric test). For each variable the difference was not significant ( $p$-value $>0.05$, ANOVA).

The distribution of the timing $t 5$ and the calculated timings cc3 and s2 were not normally distributed.

The difference was not significant for the variables $t 5$ and s2 ( $p$-value $>0.05)$, but significant $(p$-value $<0.05)$ for the calculated timing variable cc3 (Kruskall-Wallis test).

The kinetic parameter informative for embryo competence to implant (IMP-MKP) is shown in Fig. 3.

\section{Discussion}

This study defined two sets of morphokinetic parameters that are a) predictive of the embryo capacity to continue in vitro development and to become a viable blastocyst on day 5 (IVD-MKPs: t1, t2, t4, t7, t8, tC-tF, s3) and b) predictive of the embryo competence to implant and produce a viable pregnancy after day 5 transfers (IMP-MKP: cc3).

The time-lapse data showed that embryos are capable to grow into viable day 5 embryos if they have t1 value between 18.4 and $30.9 \mathrm{~h}$ post-ICSI, t2 value between 21.4 and $34.8 \mathrm{~h}, \mathrm{t} 4$ value between 33.1 and $57.2 \mathrm{~h}$, t7 value between 46.1 and $73.5 \mathrm{~h}, \mathrm{t} 8$ value 46.1 and $98.5 \mathrm{~h}, \mathrm{tC}-\mathrm{tF}$ value between 7.7 and $22.9 \mathrm{~h}$ and s3 value between 0.7 and $30.8 \mathrm{~h}$. However, these in vitro development morphokinetic parameters (IVD-MKP) are not predictive for implantation. In fact, there was no statistical difference for the $\mathrm{t} 1, \mathrm{t} 2, \mathrm{t} 4, \mathrm{t} 7$, $\mathrm{t} 8, \mathrm{tC}-\mathrm{tF}$, and $\mathrm{s} 3$ values, between embryos from the groups A-Imp (all implanted) and B (none implanted).

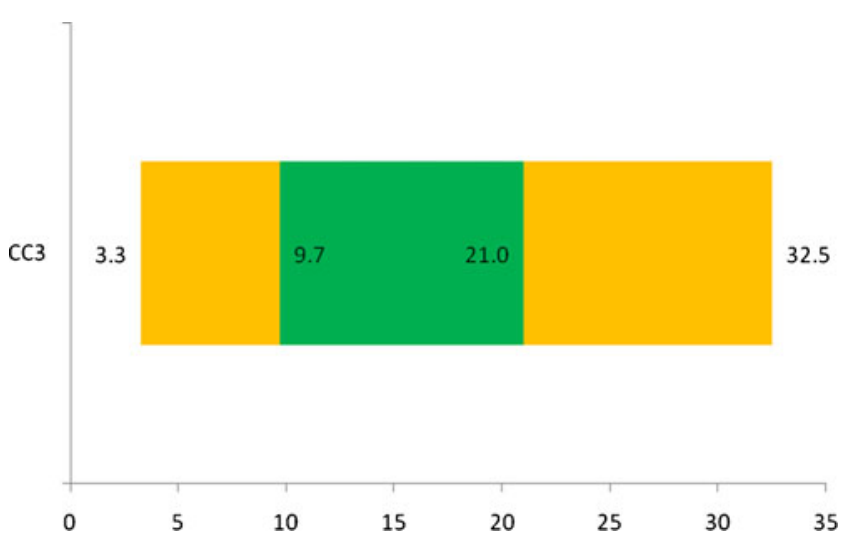

Fig. $3 \mathrm{CC} 3$ value of competent day 5 embryo to implant (Group AImp: Green) and embryos that fail to implant if transferred on day 5 (Group B: Orange). The kinetic parameters are expressed in hour and fraction of an hour
The morphokinetic parameter that was found to be significantly associated with implantation was the $\mathrm{cc} 3$. A wider amplitude of cc3 values in group B (up to $32.5 \mathrm{~h}$ ) as opposed to group A-Imp (as represented in Fig. 3) appeared to be a valid parameter to deselect embryos for transfer. From our sample, day 5 embryos with a cc3 value comprised between $9.7 \mathrm{~h}$ and $21 \mathrm{~h}$ are the ones with the highest probability to implant and produce a clinical pregnancy (IMP-MKP). Embryos with poor prognosis for in vitro development (A-NU) have a mean cc3 value of wider amplitude when compared to the cc3 of group A-imp.

In our laboratory and according to the results reported in this study, we maintain embryos in in vitro culture for a day 5 transfer only if both IVD-MKPs and IMP-MKP are in the green zone as shown in Figs. 2 and 3. If no embryos on day 3 of in vitro growth match with IVD-MKP's, a day 3 embryo-transfer is preferred. As complementary data, in all embryo-transfers with a number of implanted embryos inferior to the number of transferred embryos, the number of transferred embryos matching with IMR-MKP was superior or equal to the number of implanted embryos.

To be precise, the green intervals of time shown in Figs. 2 and 3 (in green color) should be enlarged on left side of $0.3 \mathrm{~h}$ because our time-lapse monitoring system was set to take a photogram every $20 \mathrm{~min}$ and one specific event could start to be visible $1 \mathrm{~s}$ after the previous photogram where the event still not observed.

Our results are easy to use and our method of selection excludes no competent embryo. The IVD-MKPs and IMPMKP reported here should facilitate the identification of blastocyst(s) for transfer with the highest likelihood of producing a clinical pregnancy and at the same time reduce the risk of multiple pregnancies. In addition, these parameters may be helpful to predict, as early as days 2 and 3, which embryo, if any, has the potential to grow into a viable blastocyst.

The data set of this study regarding IVD-MKP and IMPMKP were compared with those found in the literature as shown in Table 3. Our results revealed a wide variation of IVD-MKPs and IMP-MKPs among the different studies in literature. These differences might be related to in vitro culture conditions (medium, gas, etc.,) to the day of embryo transfer (day 3 or day 5) and to different patient's population (Meseguer reference of oocyte donors). Wale and Gardner [15] demonstrated that the exposure of mouse embryos to atmospheric oxygen leads to a delay of $0.45 \mathrm{~h}, 0.84 \mathrm{~h}$ and $3.19 \mathrm{~h}$ of the respective first, second and third cleavage times compared to a reduced oxygen concentration. The percentage of mouse blastocyst formation was also decreased in atmospheric oxygen. In the present work, we carried out a detailed analysis of morphokinetic development particularly for the times of PN appearance and fading and t1. Azzarello et al. [3] found that live birth event is 
Table 3 Comparison of our data and data in the literature

\begin{tabular}{|c|c|c|c|c|c|c|c|c|}
\hline \multirow{3}{*}{$\begin{array}{l}\text { MKP } \\
\text { Type of data }\end{array}$} & \multirow{3}{*}{$\begin{array}{l}\text { Our data } \\
\text { IVD/IMP* } \\
\text { Mean/Median } \\
\text { (cc3) }\end{array}$} & \multirow{3}{*}{$\begin{array}{l}\text { Azzarello } \\
\text { et al. [3] } \\
\text { IMP } \\
\text { Inferior } \\
\text { limit }\end{array}$} & \multirow{2}{*}{\multicolumn{2}{|c|}{$\begin{array}{l}\text { Ciray et al. [4] } \\
\text { IMP }\end{array}$}} & \multirow{3}{*}{$\begin{array}{l}\text { Meseguer } \\
\text { et al. [10] } \\
\text { IMP } \\
\text { Mean }\end{array}$} & \multicolumn{2}{|c|}{ Dal Canto et al. [6] } & \multirow{3}{*}{$\begin{array}{l}\text { Kirkegaard } \\
\text { et al. [8] } \\
\text { IVD } \\
\text { Median } \\
(95 \% \mathrm{CI})\end{array}$} \\
\hline & & & & & & \multirow{2}{*}{$\begin{array}{l}\text { IVD } \\
\text { Mean }\end{array}$} & \multirow{2}{*}{$\begin{array}{l}\text { IMP } \\
\text { Mean }\end{array}$} & \\
\hline & & & Mean & Mean & & & & \\
\hline $\mathrm{n}$ emb. & 72 & 37 & 9 & 9 & 61 & 93 & 19 & 53 \\
\hline $\mathrm{tC}-\mathrm{tF}$ & $15.1(7.7-22.9)$ & - & - & - & - & - & & - \\
\hline $\mathrm{t} 1$ & $24.1(18.4-30.9)$ & 20.75 & - & - & - & - & & - \\
\hline $\mathrm{t} 2$ & $26.9(21.4-34.8)$ & - & $24.7 \pm 3.6$ & $27.9 \pm 3.4$ & $25.6 \pm 2.2$ & $27.9 \pm 4.1$ & & $26.1(25.2-26.9)$ \\
\hline $\mathrm{t} 3$ & $38.3(27.8-47.8)$ & - & $35.7 \pm 5.1$ & $40.1 \pm 4.1$ & $37.4 \pm 2.8$ & $37.9 \pm 4.6$ & & $35.7(34.3-37.3)$ \\
\hline $\mathrm{t} 4$ & $40.3(33.1-57.2)$ & - & $36.6 \pm 4.3$ & $41.4 \pm 4.5$ & $38.2 \pm 3.0$ & $40.2 \pm 5.8$ & & $37.6(35.9-39.3)$ \\
\hline t5 & $53.0(37.5-67.0)$ & - & $47.4 \pm 7.9$ & $53.9 \pm 6.0$ & $52.3 \pm 4.2$ & $50.2 \pm 6.7$ & $49.4 \pm 4.9$ & $49.6(47.3-52.0)$ \\
\hline t6 & $54.3(46.1-73.5)$ & - & - & - & - & $53.9 \pm 7.6$ & & $52.4(50.1-54.9)$ \\
\hline t7 & $57.4(46.1-82.5)$ & - & - & - & - & $58.8 \pm 10.4$ & & $58.0(55.5-60.5)$ \\
\hline $\mathrm{t} 8$ & $61.0(46.4-97.8)$ & - & - & - & - & $65.2 \pm 13.0$ & $54.9 \pm 5.2$ & $60.5(57.8-63.4)$ \\
\hline $\mathrm{cc} 2$ & $11.4(1.3-14.7)$ & - & $11.1 \pm 2.4$ & $12.2 \pm 1.1$ & $11.8 \pm 1.2$ & $10.6 \pm 1.8$ & & - \\
\hline $\operatorname{cc} 3$ & $14.4(9.7-21.0)^{*}$ & - & - & - & - & - & & - \\
\hline s2 & $2.0(0-15.7)$ & - & $0.9 \pm 1.5$ & $1.2 \pm 1.3$ & $0.78 \pm 0.73$ & $2.3 \pm 3.6$ & & - \\
\hline s3 & $8.0(0.7-30.8)$ & - & - & - & - & $14.9 \pm 10.7$ & & - \\
\hline $\mathrm{CO} 2 \%$ & 5 & 5.5 & 6 & 6 & 5 & 6 & & 6 \\
\hline $\mathrm{O} 2 \%$ & 5 & 5 & 5 & 5 & 20 & 5 & & 20 \\
\hline Medium & SAGE & $\mathrm{COOK}$ & Irvine (single) & Irvine (Seq) & SAGE & Origio & & $\mathrm{COOK}$ \\
\hline Day of transfer & 5 & 2 & 4 & 4 & 3 & 3 & & - \\
\hline
\end{tabular}

IVD in vitro development until day 5; Single single step medium; Seq sequential medium

All data are expressed in hour and fraction of an hour. The significant data are in bold

associated with pronuclei breakdown not earlier than $20.75 \mathrm{~h}$ post-ICSI. Our data are in disagreement because an aliquot of embryos from group A-Imp with $\mathrm{t} 1<20.45 \mathrm{~h}$ implanted.

The study of Dal Canto et al. [6] and our own are the first to report morphokinetic parameters informative for embryos to remain viable on day 5 within the first three days of in vitro culture. We confirmed $\mathrm{t} 7, \mathrm{t} 8, \mathrm{~s} 3$ and added $\mathrm{tC}-\mathrm{tF}, \mathrm{t} 1, \mathrm{t} 2$ and $t 4$ to be predictive of IVD-MKPs. Based on a higher number of implanted embryos analyzed (19 in Dal Canto's study versus 72 in the present one), we found cc3 to be a valid morphokinetic parameter predictive of implantation. It must be reminded that the embryo starts to express its own genome at 8-cells stage and development arrests are known to occur at this point thus making cc 3 depending on $t 8$.

With time-lapse embryo monitoring it is possible to appreciate aspects of embryo development, such as duration of each cell cycle and synchronicity of cell divisions, which are not well defined in the scientific literature. The first embryonic event, pronuclei fading, appears constant in time independently of the embryonic potentiality to implant. In our culture conditions, up to $83.4 \%$ of the two pronuclei are seen between 18.5 and $19.5 \mathrm{~h}$ post ICSI.
The phenomenon of division of one to three cells can only be appreciated on dynamic observation and occurs with a frequency of $12.2 \%$ in our laboratory. These embryos are often without cellular fragments and with blastomeres regular in symmetry and size. Based solely on conventional microscopy (observation at $42 \mathrm{~h}$ ), it is easy to miss embryos with asynchronous division. Rubio et al. [14] showed that embryos with cc2 shorter than $5 \mathrm{~h}$ have a reduced potential to implant. It is interesting to note that from the 5 cell-stage, the embryo tends to lose the cell synchronicity. The transition of a 5-cells stage embryo to 8-cells stage takes around $8 \mathrm{~h}$ in implanting embryos (s3).

It was also possible to observe that $6.9 \%$ and $4.4 \%$ of 2 cells stage embryos had one or two multi-nucleated cells (11.3\%. of total multi-nucleation). The presence of multinucleated blastomeres in human embryos is correlated with chromosomal abnormalities [9] and the nucleus in the binucleated cells is of androgenic origin [18].

High frequencies of abnormal events during embryonic development must be known, and their biological meaning in term of embryo competence must be understood. The traceability of transferred embryos to asynchronous divisions and/or multi-nucleation at early stages show that they can implant and give healthy babies even if the implantation 
rate seems lower than embryos without these characteristics. A vacuole present from the 1-cell stage can be isolated to trophectoderm cells along the cell divisions. As a consequence, it appears evident that the embryo has the capability to repair itself. Similarly, an asynchronous division could be a phenomenon of discarding cellular material because it should not be part of what will later be the inner cell mass. Further studies with time-lapse are needed to evaluate the competence of embryos with specific morphological characteristics.

Because of the wide variation of IVD-MKPs and IMPMKPs informative in the scientific literature, we recommend to each laboratory to determine its own IVD-MKP and IMP-MKP selective in its conditions of work or to consider only the published studies that work in exactly the same conditions (medium, gas...).

In conclusion, time-lapse monitoring gives the possibility to assess the human embryo during the various dynamic developmental activities and to establish kinetic parameters predictive of embryonic competence. The additional embryonic events observable in time-lapse monitoring compared to normal microscopy, suggest the necessity to elaborate a new embryo scoring that must reflect embryo quality as it is currently annotated plus multi-nucleation, asynchronous divisions at a specified cell-stage and the kinetic of embryo development.

Open Access This article is distributed under the terms of the Creative Commons Attribution License which permits any use, distribution, and reproduction in any medium, provided the original author(s) and the source are credited.

\section{References}

1. Alpha Scientists in Reproductive Medicine and ESHRE Special Interest Group of Embryology. The Istanbul consensus workshop on embryo assessment: proceedings of an expert meeting. Hum Reprod. 2011;26(6):1270-83.

2. Arav A, Aroyo A, Yavin S, Roth Z. Prediction of embryonic developmental competence by time-lapse observation and 'shortest-half' analysis. Reproductive BioMedicine on line. 2008;17(5):669-75.

3. Azzarello A, Hoest T, Mikkelsen AL. The impact of pronuclei morphology and dynamicity on live birth outcome after time-lapse culture. Hum Reprod. 2012;27(9):2649-57.
4. Ciray HN, Aksay T, Goktas C, Ozturk B, Bahceci M. Time-lapse evaluation of human embryo development in single versus sequential culture media - a sibling oocyte study. J Assist Reprod Genet. 2012;29(9):891-900.

5. Cruz M, Gadea B, Garrido N, et al. Embryo quality, blastocyst and ongoing pregnancy rates in oocyte donation patients whose embryos were monitored by time-lapse imaging. J Assist Reprod Genet. 2011;28(7):569-73.

6. Dal Canto M, Coticchio G, Mignini Renzini M, et al. Cleavage kinetic analysis of human embryos predicts development to blastocyst and implantation. Reproductive BioMedicine Online. 2012 on publication.

7. Guerif F, Lemseffer M, Leger J, Bidault R, Cadoret V, Chavez C, et al. Does early morphology provide additional selection power to blastocyst selection for transfer. Reproductive BioMedicine Online. 2010;21:510-9.

8. Kirkegaard K, Hindkjaer JJ, Ingerslev HJ. Human embryonic development after blastomere removal: a time-lapse analysis. Hum Reprod. 2012;27(1):97-105.

9. Kligman I, Benadiva C, Alikani M, Munné S. The presence of multinucleated blastomeres in human embryos is correlated with chromosomal abnormalities. Hum Reprod. 1996;11(7):1492-8.

10. Meseguer M, Herrero J, Tejera A, Hilligsøe KM, Ramsing NB, Remohí J. The use of morphokinetics as a predictor of embryo implantation. Hum Reprod. 2011;26(10):2658-71.

11. Nel-Themaat L, Nagy ZP. A review of the promises and pitfalls of oocyte and embryo metabolomics. Placenta. 2011;32:S257-63.

12. Pribenszky C, Losonczi E, Molnar M, et al. Prediction of in-vitro development competence of early cleavage-stage mouse embryos with compact time-lapse equipment. Reproductive BioMedicine Online. 2010a;20:371-9.

13. Pribenszky C, Mátyás S, Kovács P, Losonczi E, Zádori J, Vajta G. Pregnancy achieved by transfer of a single blastocyst selected by time-lapse monitoring. Reproductive BioMedicine Online. 2010b;21(4):533-6.

14. Rubio I, Kulmann R, Aherholm I et al. Limited implantation success of direct-cleaved human zygote: a time-lapse study. Fertility and Sterility. 2012 On press.

15. Wale PL, Gardner DK. Time-lapse analysis of mouse embryo development in oxygen gradient. Reproductive Biomedicine Online. 2010;21:402-10.

16. Wong C, Loewke KE, Bossert NL, et al. Non-invasive imaging of human embryos before embryonic genome activation predicts development to the blastocyst stage. Nat Biotechnol. 2010;28(10):111524.

17. Wong C, Chen AA, Behr B, Shen S. Time-lapse microscopy and image analysis in basic and clinical embryo development development research. Reproductive BioMedicine On Line. 2013;26:120-9.

18. Xanthopoulou L, Delhanty JDA, Mania A, et al. The nature and origin of binucleate cells in human preimplantation embryos: relevance to placental mesenchymal dysplasia. Reproductive BioMedicine Online. 2011;22:362-70. 